\title{
Atomic Force Microscopy Investigation of the
}

\author{
Morphology and the Biological Activity of
}

\section{Protein-Modified Surfaces for Bio- and}

\section{Immuno-Sensors}

Francesca Cecchet, Anne-Sophie Duwez, Sabine Gabriel, Christine Jérôme, Robert Jérôme, Karine Glinel, Sophie Demoustier-Champagne, Alain M. Jonas and Bernard Nysten

\section{AFM single molecule force spectroscopy}

\section{Histogram of the rupture distances}

Figure SI1 shows the histograms of the tip-sample distances at which the corresponding unbinding events have been observed for both protein systems. The fit with a Gaussian profile gives a most probable tip-sample distance of $48 \mathrm{~nm}$ for the $69 \mathrm{~K} \mathrm{Ag}$ system and of $18 \mathrm{~nm}$ for $286 \mathrm{~F} 7 \mathrm{Ab}$. Since the $\mathrm{Ab}$ or $\mathrm{Ag}$ is linked at a specific position on the linker, the distribution of the distances at which the rupture forces are observed is relatively narrow. This is not the case for non-specific events, which may appear at any distance, giving rise to very broad distributions. The appearance of rupture peaks at a certain distance is a classical criterion widely used to distinguish between specific and non-specific events. 

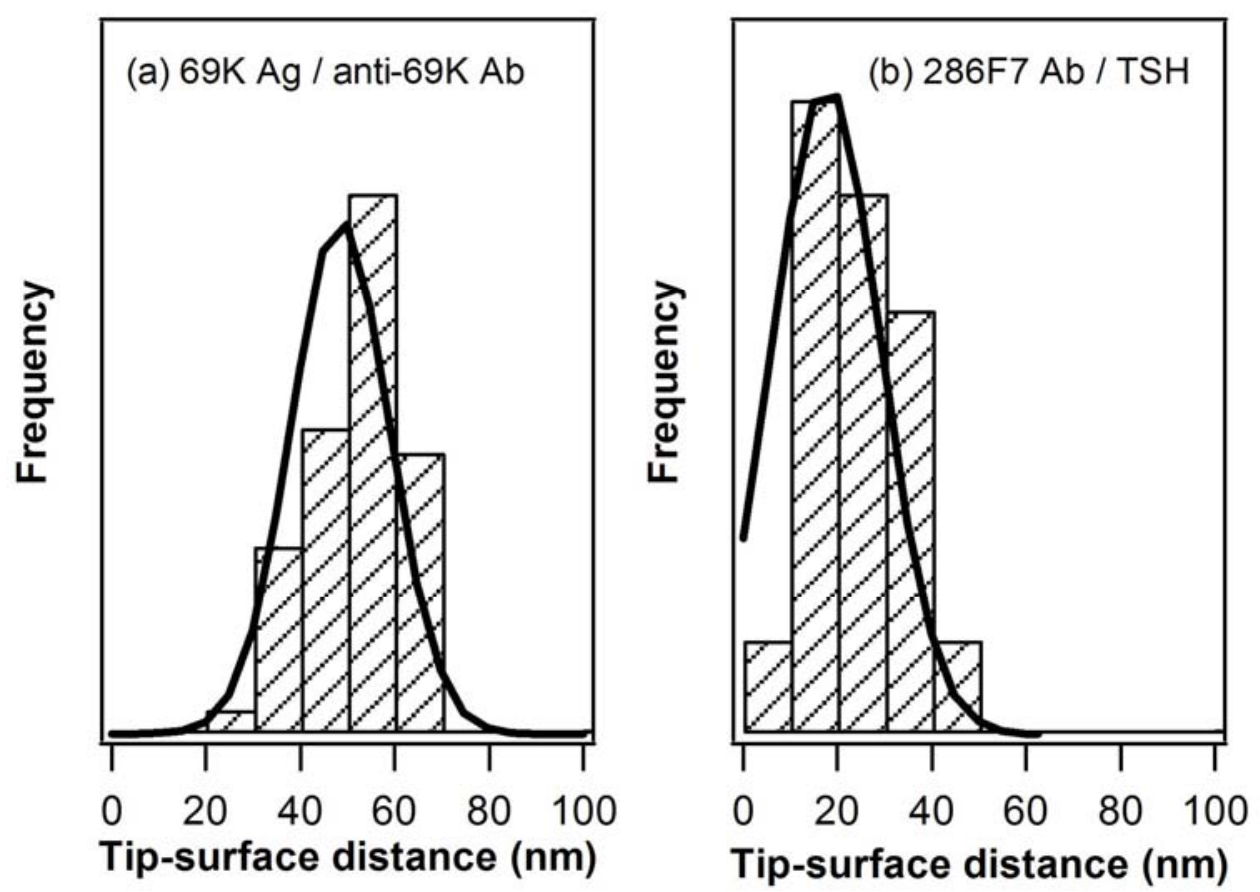

Figure SI1. Distribution of the distances at which the rupture forces have been observed for the $69 \mathrm{~K} \mathrm{Ag}$ system (a) and for the 286F7 Ab system (b).

\section{Loading-rate dependent measurements}

A dynamic force spectroscopy investigation has been carried out for both protein systems, by varying the loading rate over three orders of magnitude, from $510^{2} \mathrm{pN} / \mathrm{s}$ up to $110^{6} \mathrm{pN} / \mathrm{s}$.

In figure SI2 the measured unbinding forces (obtained from the most probable value resulting from the fitting of the force histogram at each loading rate) are plotted as a function of the logarithm of the loading rate. We observe that for both protein systems the relationship between the force and the loading rate is characterized by two regimes, each one showing a linear increase of the force with the loading rate. The two distinct linear regimes suggest the existence of two energy barriers along the reaction coordinates [Evans, E., Annu. Rev. Biophys. Biomol. Struct. 2001, 30, 105-128]. Each regime is governed by the relation expressed in equation 1

$$
F=\frac{k_{B} T}{x_{\beta}} \ln \left(\frac{x_{\beta} r}{k_{\text {off }} k_{B} T}\right)
$$


where $k_{B}$ is the Boltzmann constant, $T$ the temperature, $x_{\beta}$ is the width of the potential of the bound state and $k_{\text {off }}$ is the thermal off-rate. Hence, the fitting of each regime with equation (1) gives an estimation for the thermal off-rate from the extrapolation to zero unbinding force. The fitted values are reported in Figure SI1 for each linear regime. Those values should be considered with caution. Systematic studies on nonlinear loading effects of the linker, which might distort the energy landscape, are underway.
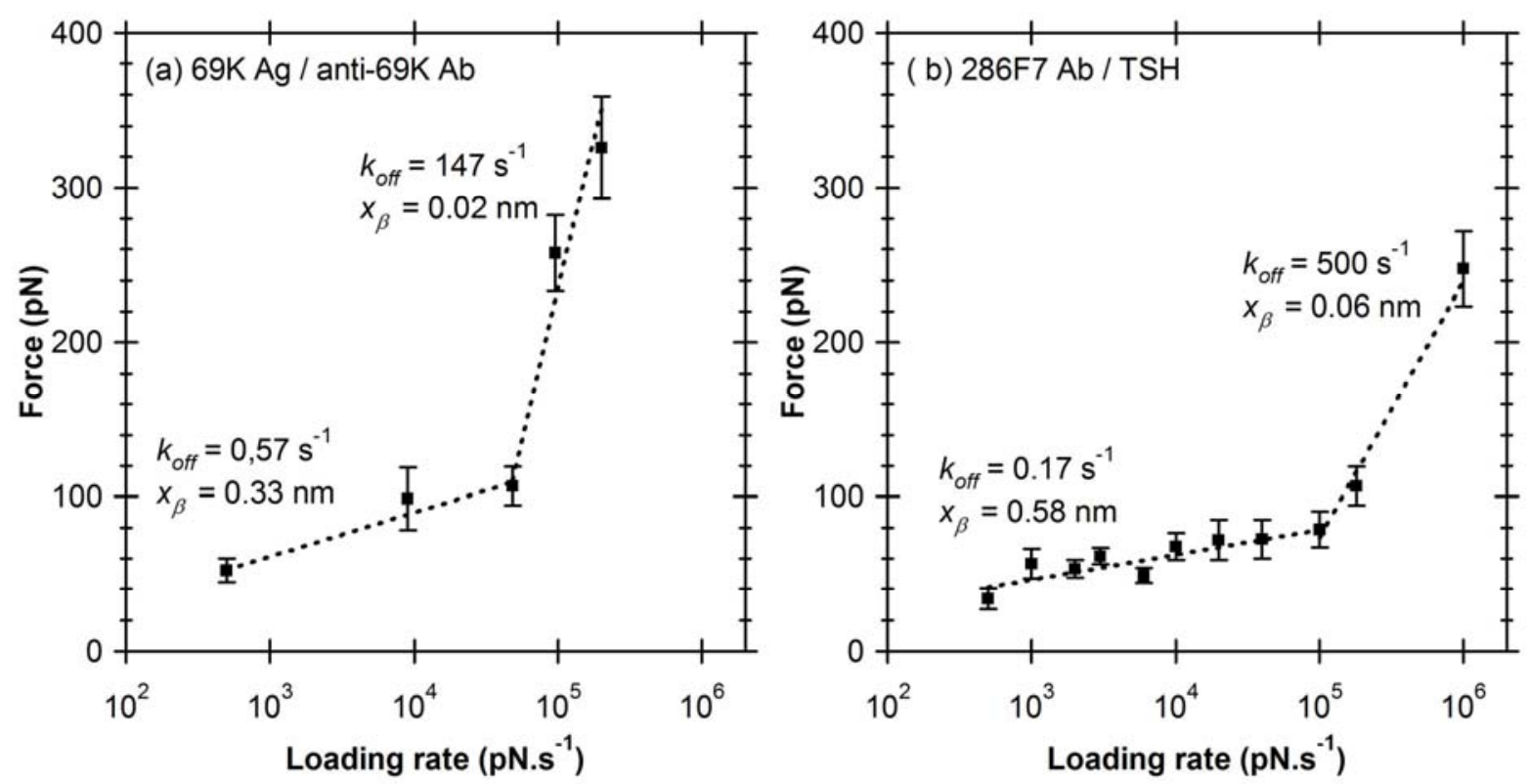

Figure SI2: Loading rate dependence of the unbinding forces for $69 \mathrm{~K} \mathrm{Ag} / \mathrm{anti}-69 \mathrm{~K} \mathrm{Ab}$ (a) and for 286F7 Ab / TSH hormone (b), at room temperature. The error of the unbinding forces was estimated as the width at half-maximum of the Gaussian fit of the unbinding force distributions. The off-rates at zero force, $k_{\text {off, }}$ and the molecular lengths (along the reaction coordinates), $x_{\beta n}$ have been determined from linear fits to the data with equation 1. 